\title{
Conhecimentos atuais em Fissuras Labiopalatinas: uma revisão narrativa
}

\author{
Current Knowledge in Cleft lip and palate: a narrative review
}

\author{
Conocimiento actual en Fisuras Labiopalatinas: una revisión narrativa \\ Eliane Alves Motta Cabello dos Santos ${ }^{1 *}$, Thais Marchini de Oliveira ${ }^{1}$.
}

\begin{abstract}
RESUMO
Objetivo: Realizar revisão crítica da literatura abrangendo os aspectos etiológicos, epidemiológicos, diagnósticos e de tratamento em fissuras labiopalatinas (FLP). Revisão bibliográfica: As FLP representam as malformações congênitas craniofaciais mais prevalentes mundialmente. A etiologia das FLP é complexa e multifatorial, envolvendo a susceptibilidade genética e a exposição materna aos fatores de risco ambientais. Clinicamente, os indivíduos acometidos apresentam problemas de fala, alimentação, audição e de interação social. Recomenda-se tratamento interdisciplinar e especializado, que pode se prolongar do nascimento à idade adulta. Considerações finais: $A$ FLP é uma condição para toda a vida, e acarreta problemas que vão além do complexo craniofacial. Apesar dos grandes avanços na compreensão da FLP, algumas questões permanecem pouco estudadas, como é o caso das comorbidades não diretamente relacionadas à malformação e seu impacto ao longo da vida. A maioria dos autores ressaltam a importância da avaliação precoce da equipe interdisciplinar, do acompanhamento ao longo prazo e das pesquisas, para o manejo correto do paciente com FLP.
\end{abstract}

Palavras-chave: Fissura palatina, Fenda labial, Epidemiologia, Reabilitação.

\section{ABSTRACT}

Objective: The aim of the current study was to review the epidemiology, the etiology and the management of cleft lip and/or palate (CLP). Literature review: CLP are among the most common congenital anomalies affecting the head and neck. The etiology of FLP is complex and multifactorial and includes genetic susceptibility and environmental risk factors. Clinically, the patients may have problems with speech, feeding, impaired hearing and difficulties in social interaction. Interdisciplinary and specialized treatment is recommended, from birth to adulthood. Final considerations: FLP is a lifelong condition and may lead to problems beyond the craniofacial complex. Despite current advances in the knowledge on CLP, some issues remain unclear, such as the comorbidities not directly related to the malformation and the longer-term impact of CLP. Researching on CLP, early interdisciplinary evaluation, and long-term follow-up of patients with orofacial clefting are essential to achieving optimal clinical outcomes.

Keywords: Cleft lip, Cleft palate, Epidemiology, Rehabilitation.

\section{RESUMEN}

Objetivo: Realizar una revisión crítica de la literatura acerca de los aspectos etiológicos, epidemiológicos, diagnósticos y terapéuticos del labio leporino y de la fisura palatina. Revisión bibliográfica: El labio leporino y el paladar hendido representan las anomalías congénitas craneofaciales más prevalentes en el mundo. La etiología es compleja y multifactorial, e involucra susceptibilidad genética y exposición materna a factores de riesgo ambientales. Clínicamente, los individuos afectados tienen dificultades con el habla, la alimentación, la audición e la interacción social. Se recomienda un tratamiento interdisciplinario y especializado, desde el nacimiento hasta la edad adulta. Consideraciones finales: El labio leporino y la hendidura del paladar son condiciones de por vida y causan problemas más allá del complejo craneofacial. A pesar de los grandes avances en la comprensión del tema, las comorbilidades no relacionadas directamente con la malformación y su impacto a lo largo de la vida siguen siendo poco estudiados. La mayoría de los autores enfatizan la importancia de la evaluación temprana por parte del equipo interdisciplinario, el seguimiento e investigación a largo plazo, para el correcto manejo de los pacientes.

Palabras Clave: Labio leporino, fisura del paladar, epidemiología, rehabilitación.

\footnotetext{
${ }^{1}$ Hospital de Reabilitação de Anomalias Craniofaciais, Universidade de São Paulo (USP), Bauru - SP.

*E-mail: elianecabello@usp.br
} 


\section{INTRODUÇÃO}

As Fissuras Labiopalatinas (FLP) representam as malformações congênitas craniofaciais mais prevalentes mundialmente, com média estimada em 1/700 nascidos vivos (DIXON MJ, et al., 2011). Afetam estrutural e funcionalmente a face e a cavidade oral, levando a repercussões clínicas que requerem cuidados do nascimento à idade adulta (LEWIS CW, et al., 2017). Os custos financeiros e emocionais para o adequado processo de reabilitação podem ser consideráveis, sendo especialmente desafiadores aos países em desenvolvimento (VOLK AS, et al., 2020).

Segundo a apresentação fenotípica, as FLP se manifestam como Fissura de Palato (FP), acometendo palato isoladamente, Fissura de Lábio $(\mathrm{FL})$ isolada e $\mathrm{FL}$ associada à $\mathrm{FP}$, unilaterais ou bilaterais (DIXON MJ, et al., 2011). A malformação se desenvolve durante o período embrionário de formação das estruturas da face e palato, da quarta à décima segunda semana de vida intrauterina (FREITAS JAS, et al., 2012).

Falhas nas linhas de fusão dos processos primordiais da face, levariam aos espaços congênitos anormais, também denominados fissuras (FREITAS JAS, et al., 2012). Cerca de $30 \%$ dos casos de FLP são associados a síndromes, mas a maioria das FLP são não sindrômicas e têm etiologia multifatorial (BURG ML, et al., 2016).

Uma complexa interação entre fatores genéticos e exposição materna aos fatores ambientais ou epigenéticos, ainda pouco compreendidos, são as causas possíveis (MURRAY J et al., 2002). Esforços têm sido realizados no sentido de associar determinados genes e variáveis ambientais, como deficiências nutricionais, uso de drogas e medicações ao desenvolvimento das FLP (MANGOLD E, et al., 2010; GILDESTAD T, et al., 2015).

Clinicamente, as FLP levam a problemas físicos anatômicos e funcionais, além de problemas emocionais ao paciente (LEWIS CW, et al, 2017). O acometimento do palato associa-se às manifestações clínicas mais evidentes, como os distúrbios alimentares que podem afetar um terço das crianças com FLP (BOYCE JO, et al., 2019; REID J, et al., 2006). Ainda, alterações da fala por disfunção do mecanismo velofaríngeo e alterações de audição secundárias às otites médias por efusão de repetição são comuns (HOSSEINBAD HH, et al, 2015).

Problemas psicossociais pessoais e familiares são relatados, afetando a interação social e o desempenho escolar do paciente (NELSON PA, et al., 2012; WHEBY G, et al., 2012). Alguns autores ressaltam a ocorrência de sintomas que atingem outros sistemas orgânicos além do complexo craniofacial, como anemias, alguns tipos de câncer e problemas de saúde mental na idade adulta (SILVA HPV, et al, 2018; CRHISTENSEN K, et al., 2004). O tratamento do paciente com FLP constitui um desafio, pois requer uma equipe interdisciplinar treinada e altamente especializada com capacidade e disponibilidade para atender às necessidades físicas $\mathrm{e}$ emocionais da família e do indivíduo com FLP (LEWIS CW, et al., 2017).

Os protocolos de tratamento clínico e cirúrgico divergem entre os serviços, mas é consenso que o diagnóstico precoce e o acompanhamento em serviço especializado com profissionais médicos, dentistas, fonoaudiólogos, geneticistas, nutricionistas e enfermeiros, são altamente recomendados (CHEPLA KJ e GOSAIN AK, 2013; VOLK AS, et al., 2020). O manejo cirúrgico inclui as cirurgias primárias na infância como queiloplastia e palatoplastia, as cirurgias secundárias, os enxertos osteoalveolares, além das cirurgias ortognáticas na adolescência (FREITAS JAS, et al., 2012).

$\mathrm{O}$ cuidado oferecido aos indivíduos com FLP deve seguir os protocolos de segurança e qualidade recomendados pela Organização Mundial de Saúde (TRINDADE IEK e SILVA FILHO OG, 2007). Assim, os serviços públicos voltados para a pesquisa e atendimento ao paciente com fissura, aliados a projetos de parceria internacional, como é o caso do Programa "Smile Train", são iniciativas que efetivamente contribuem para a melhoria nas condições de vida do indivíduo com FLP e seus familiares (VOLK AS, et al., FREITAS JAS, et al., 2012).

O objetivo do presente estudo foi o de realizar uma revisão crítica da literatura abrangendo os aspectos etiológicos, epidemiológicos, diagnósticos e de tratamento em FLP, visando auxiliar na compreensão deste importante problema de saúde pública. 


\section{REVISÃO BIBLIOGRÁFICA}

\section{Aspectos epidemiológicos}

Há variações na prevalência de FLP de acordo com aspectos geográficos e étnicos da população estudada. Os asiáticos e nativos americanos costumam ter as maiores prevalências (cerca de 1/500 nascidos vivos), enquanto os povos africanos têm as taxas menores (1/2500) (DIXON MJ, et al., 2011). Entre os estados brasileiros, observa-se os maiores percentuais no sudeste do país e os menores nos estados do nordeste (SOUZA GF e RONCALLI AG, 2017).Há predominância de prevalência geral das FLP no sexo masculino, na proporção de 2:1 enquanto as fissuras isoladas de palato (FP) prevalecem em meninas (DIXON MJ, et al., 2011).Os tipos mais comuns de FLP são as que envolvem concomitantemente o palato primário e o secundário (FREITAS JAS, et al., 2012).

\section{Embriologia}

O desenvolvimento das fissuras labiopalatinas acompanha a formação da face e do palato e decorre da ação dos Fatores Teratogênicos (FT) genéticos e epigenéticos sobre as linhas de fusão dos processos faciais primordiais (FREITAS JAS, et al., 2012). A fusão dos processos maxilares, mandibulares e fronto-nasal, é gradual, da quarta até a oitava semana de vida intrauterina, quando o embrião adquire feições humanas. Neste período de quatro semanas, falhas nas linhas de fusão resultarão na formação das fissuras de lábio e de rebordo alveolar (FREITAS JAS, et al., 2012). O palato concluirá seu processo de fusão da sexta à décima segunda semana, e é neste intervalo que poderá se instalar a fissura de palato (TRINDADE IEK e SILVA FILHO OG, 2007).

\section{Etiologia}

Aproximadamente $70 \%$ das FLP são isoladas e não se associam a outras anomalias ou Síndromes (DIXON MJ, et al., 2011). Nos casos de fissura isolada de palato (FP), no entanto, a proporção de síndromes associadas se eleva para cerca de $50 \%$. São conhecidas mais de 500 síndromes associadas às FLP, que podem ser do tipo Mendelianas, secundárias a alterações cromossômicas ou ainda, teratogênicas. Exemplos incluem as síndromes de Apert, de CHARGE, de Treacher Collins e outras (BURG ML, et al., 2016).

\section{Fatores de risco genéticos e ambientais associados ao desenvolvimento de FLP não sindrômicas.}

História familiar positiva é comum aos pacientes com FLP (GROSEN D, et al., 2010). Pais com um filho com FLP terão chance de 2 a $5 \%$ de que o próximo filho também seja afetado. Essas observações sugerem o envolvimento de herança genética na etiologia das FLP. Muitos esforços têm sido realizados para a identificação de genes relacionados às FLP. Estudos com mapeamento genético, com genes candidatos e ainda, através da extrapolação dos dados obtidos em estudos de casos sindrômicos têm sido realizados (MANGOLD E, et al., 2010). Assim, têm se estabelecido associações de determinados genes ao desenvolvimento das FLP, como por exemplo, o Interferon Regulatory Factor 6 (IRF6), o Transforming Growth Factor Alpha (TGFA) e o Fibroblast Growth Factor Receptor 2 (FGFR2).

No entanto, as evidências apontam para o modelo de herança multifatorial, que envolve os fatores de risco genéticos interagindo com variáveis ambientais (DIXON MJ, et al., 2011). O papel causal dos teratógenos na formação de fissuras têm sido esclarecidos em estudos da exposição materna a fatores como álcool, tabaco, uso de corticóides, de ácido valpróico e de ácido retinóico (MURRAY J, et al., 2002). Interações específicas genético ambientais aumentam o risco de formação de FLP não sindrômica. O tabagismo materno durante a gravidez dobra o risco de FLP e mulheres fumantes que apresentam algumas variações genéticas específicas (como nos genes GSTT1 e NOS3) terão um risco ainda maior (SHI M et al., 2007).

A exposição ao álcool através do consumo materno na gestação é outro fator de risco conhecido e o consumo de altas doses por curtos períodos, associado a variações no gene ADH1C aumentará ainda mais a prevalência de FLP (DeROO LA, et al., 2016). Fatores nutricionais como deficiência de folatos e de zinco podem influenciar o risco de desenvolvimento de FLP. A suplementação de ácido fólico no pré-natal leva à diminuição da prevalência de FLP associadas a outras malformações (GILDESTAD T, et al., 2015). Assim, recomendação de suplementação de ácido fólico para gestantes, previne problemas como espinha bífida, anencefalia e ainda se associa à diminuição da prevalência de alguns tipos de FLP (LEWIS CW, et al 2017). 
Drogas anticonvulsivantes na gestação que interferem com o metabolismo dos folatos, assim como hipertermia, diabetes, obesidade materna, estresse, radiação ionizada e infecções também são considerados como fatores de risco para FLP não sindrômicas (MOSSEY PA, et al; 2009).

\section{Classificação morfológica}

Historicamente a FLP é dividida FP isolada, FL isolada e FL associada a FP, no entanto, a expressão fenotípica é mais complexa e outros modelos de classificação são utilizados por clínicos e pesquisadores (DIXON MJ, et al., 2011; TRINDADE IEK e SILVA FILHO OG, 2007). Alguns centros especializados nacionais adotam a Classificação de SPINA modificada por Silva Filho. Trata-se de um sistema de classificação com base na embriologia e que utiliza como referência o Forame Incisivo (FI), vestígio da estrutura embrionária que separava o palato primário do palato secundário na vida intrauterina (FREITAS JAS, et al., 2012).

Assim, as fissuras pós-forames incisivas acometem o palato isoladamente, as fissuras transforame acometem lábio e palato e a pré-forame incisivo constitui a fissura isolada de lábio. As denominadas fissuras raras de face não têm vinculação com o FI (TRINDADE IEK e SILVA FILHO OG, 2007).

A referência ao fenótipo como a simples presença ou ausência de fissura, tem sido vista como insuficiente para o entendimento da etiologia e da epidemiologia da malformação (DIXON MJ, et al., 2011). Evidências sugerem que o espectro fenotípico deveria incluir também as manifestações subclínicas, que são alterações estruturais menores, muitas vezes presentes nos pacientes com FLP e nos familiares não aparentemente afetados (WEINBERG S, et al., 2008). Assim, alterações como a disruptura do músculo orbicular da boca (observado por ultrassonografia), presença de fossetas labiais, anomalias dentárias ou úvula bífida seriam considerados fenótipos subclínicos (WEINBERG S, et al., 2008; DIXON MJ, et al., 2011).

\section{Diagnóstico}

O diagnóstico pré-natal é possível através do exame de ultrassonografia (US). Com o adequado posicionamento da face, a FL pode ser detectada no segundo trimestre de gestação, enquanto a observação do palato é mais complexa e requer recursos tecnológicos mais sofisticados como US tridimensional (ROTTEN D e LEVAILLANT JM, 2004). Esforços para o diagnóstico pré-natal são justificáveis, pois possibilitam aos pais prepararem-se emocionalmente e obterem informações apropriadas e aconselhamento a respeito da anomalia (DIXON MJ, et al., 2011).

Não raro, a fissura é percebida apenas ao nascimento ou ainda mais tardiamente, após a alta da maternidade (LEWIS CW, et al., 2017). Os pacientes com atrasos no diagnóstico frequentemente evoluem com problemas alimentares e regurgitação nasal. Assim, a inspeção visual do palato e a palpação devem ser realizadas logo ao nascimento para checar a presença de fissuras (LEWIS CW, et al., 2017; BOYCE JO, et al., 2019).

\section{Manifestações clínicas}

Os problemas funcionais e estéticos causados pelas FLP são complexos e podem levar a manifestações clínicas e psicossociais secundárias, com importante repercussão na vida dos pacientes (MOSSEY PA, et al., 2009). Alguns fatores são determinantes para a maior intensidade das manifestações clínicas, como baixa idade, presença de fissura de palato, diagnóstico tardio e presença de outras malformações associadas (BOYCE JO, et al., 2019; MARTIN V e GREATREX-WHITE S, 2014).

Fissuras isoladas de lábio provocarão predominância de problemas estéticos, com possibilidade de alterações dentárias quando o arco alveolar é envolvido (FREITAS JAS, et al., 2012). O acometimento do palato (mesmo que isolado) se associa às manifestações clínicas mais intensas, apesar de causar menos alterações estéticas (BOYCE JO, et al., 2019).

As fissuras com comprometimento de lábio e palato unilaterais ao dividir a maxila em duas partes, levam à assimetria nasal, ruptura da musculatura perilabial e comunicação das cavidades oral e nasal. Esse espectro de alterações resultará em problemas alimentares e na fala. Indivíduos com fissuras bilaterais de lábio e palato terão segmentação maxilar em três partes, com projeção da pré-maxila, acarretando repercussões estéticas e funcionais expressivas (FREITAS JAS, et al., 2012). 


\section{Problemas alimentares}

Cerca de um terço das crianças com FLP apresenta distúrbios alimentares em decorrência da dificuldade na formação da pressão negativa intraoral necessária à sucção e deglutição (REID J, et al, 2006). Existe uma correlação entre a amplitude, o tipo de fissura, a maturidade do recém-nascido e a quantidade de pressão gerada durante a alimentação. Crianças mais novas, com fissuras mais amplas terão maiores dificuldades à alimentação, com engasgos, refluxo nasal de alimentos e tempo prolongado de alimentação e consequentemente dificuldades no ganho de peso (MARTIN V e GREATREX-WHITE S, 2014).

Especial atenção deve ser dada ao aleitamento materno, pelos inúmeros benefícios à saúde do binômio mãe e filho (BOYCE JO, et al., 2019). Há evidências de que as crianças com acometimento de palato têm maiores dificuldades à amamentação, porém, o encorajamento e orientação de práticas apropriadas ao aleitamento materno são altamente recomendadas em todos os casos (BOYCE JO, et al., 2019). Posicionamento do bebê, oclusão da fissura de lábio com o dedo, expressão da mama são algumas técnicas utilizadas. Quando insuficientes, pode ser necessária a complementação do LM ordenhado com copo, colher ou seringa. $\mathrm{Na}$ impossibilidade de aleitamento materno, mamadeiras que facilitem o fluxo de leite, com fórmulas infantis são utilizadas (BOYCE JO, et al., 2019).

Mães com problemas para alimentar seus filhos são mais propensas a depressão, dificultando ainda mais os cuidados com o bebê com FLP (MARTIN V e GREATREX-WHITE S, 2014). Ainda, na fase de introdução da dieta sólida poderá ocorrer temor por parte dos cuidadores da criança, em oferecer alimentos novos, podendo resultar em insuficiente aporte de fibras e outros nutrientes (TRINDADE IEK e SILVA FILHO OG, 2007).

\section{Distúrbios da fala e da audição}

As alterações de fala decorrentes da FLP são diversas e em grande parte relacionam-se à disfunção do mecanismo velofaríngeo. Assim, ocorrem distúrbios articulatórios do desenvolvimento, distúrbios articulatórios compensatórios, distúrbios obrigatórios (como a hipernasalidade) e adaptações compensatórias, que atrapalham a inteligibilidade da fala (HOSSEINBAD HH, et al, 2015). De 10 a 25\% dos pacientes com FLP terão persistência das dificuldades de fala, denominada insuficiência velofaríngea (IVF) após o reparo do palato (HOSSEINBAD HH, et al., 2015). Os problemas da fala devem ser acompanhados com cuidado, pois impactam negativamente em vários aspectos da vida, como socialização e desempenho escolar (LEWIS CW, et al, 2017). A Otite Média com Efusão (OME) constitui uma preocupação em crianças com FLP. Ocorre por disfunção da tuba auditiva e associa-se à perda auditiva em grande porcentagem dos casos (KUO CL et al., 2014).

\section{Problemas psicossociais pessoais e familiares}

Os pacientes com FLP enfrentam o estresse muito precocemente na vida. Procedimentos cirúrgicos, dificuldades com a fala e alimentação, insatisfação com a aparência, menor aceitação social, dificuldades escolares e bullying são frequentes fatores de estresse (WHEBY G, et al., 2012). Independentemente de idade, sexo e diferenças culturais, os indivíduos com FLP tem um ajuste psicossocial pior que os indivíduos sem FLP (HUTCHINSON K, et al., 2011). Sintomas de depressão e ansiedade são frequentemente observados em crianças com FLP (HUNT O, et al., 2005). Os adolescentes relatam experiências estigmatizantes que afetam a qualidade de vida (LEWIS CW, et al., 2017).

O estresse acomete também os pais das crianças com FLP. O nascimento de um filho com a malformação pode evocar sentimentos conflitantes, como culpa, choque e preocupações com o futuro (NELSON PA, et al., 2012). Há dificuldades em apresentar o recém-nascido aos familiares, incertezas, necessidade de preparação para as cirurgias e para o tratamento prolongado. Assim, o suporte psicológico para o enfrentamento do estresse, da ansiedade e do medo é crucial para o paciente e familiares (NELSON PA, et al., 2012).

\section{Crescimento e desenvolvimento físico}

Existem controvérsias com relação ao padrão de crescimento das crianças com FLP não sindrômicas (MARQUES IL, et al., 2009). O desenvolvimento físico insatisfatório tem relação com nutrição inadequada, problemas crônicos de saúde e doenças genéticas. 
O paciente com FLP não sindrômica poderá apresentar medidas de peso e altura menores no primeiro ano de vida, com recuperação gradual até o final do segundo ano de idade (MARQUES IL, et al., 2009). As dificuldades alimentares e doenças respiratórias podem justificar esse impacto negativo da FLP no crescimento físico.

\section{Comorbidades para além do complexo craniofacial}

A FLP representa um distúrbio de longa evolução que vai muito além do complexo craniofacial, repercutindo em vários outros sistemas do organismo e o reconhecimento deste fato possibilita a elaboração de protocolos visando proporcionar um padrão de vida mais saudável ao paciente (DIXON MJ, et al., 2011).

Estudo nacional realizado em 2018 estimou em 45,7\% a prevalência de comorbidades em pacientes com FLP, incluindo problemas como anemias $(16,2 \%)$, doenças respiratórias $(12,2 \%)$, cardíacas $(9,8 \%)$ e neurológicas $(8,1 \%)$ (SILVA HPV, et al, 2018). Metade dos pacientes com atraso concomitantemente nas áreas motora, de fala e comportamental ( $8,1 \%$ do total) não recebiam nenhuma terapia de suporte. Condições adicionais como transtorno do déficit de atenção com hiperatividade, atrasos no desenvolvimento neuropsicomotor e dislexia são também observados (FERAGEN KB, et al., 2015).

Ainda, o acompanhamento ao longo prazo de indivíduos com FLP demonstra maior risco de mortalidade, problemas de saúde mental e de câncer quando comparados aos indivíduos saudáveis (CRHISTENSEN K, et al., 2004; ZHU JL, et al., 2002). Alguns genes têm sido simultaneamente associados ao câncer e às anomalias craniofaciais, como é o caso das mutações no gene $\mathrm{CDH} 1$, observadas em famílias com história de câncer gástrico difuso e FLP (FREBOURG T, et al.,2006).

A identificação dos problemas adversos associados às FLP no decorrer da vida, não diretamente relacionados à malformação, pode resultar em melhoria das condições de saúde do paciente, por permitir o reconhecimento dos riscos em seus estágios precoces (DIXON MJ, et al., 2011, STOCK NM, et al., 2015). São poucos os estudos disponíveis sobre as repercussões da FLP em sistemas orgânicos não diretamente relacionados à malformação. Poucos também são os conhecimentos da evolução ao longo prazo do paciente adulto (STOCK NM, et al., 2015).

Ainda, o entendimento de que a FLP é uma condição para toda a vida, reforça a necessidade de mais pesquisas nos diferentes domínios interpessoais, auto estima, vida profissional e afetiva nas diversas fases da vida (STOCK NM, et al., 2015; ZEITNOGLU S e DAVEY MP, 2012). Estes estudos permitiriam identificar os fatores que contribuem para o estresse psicológico e resiliência, bem como os períodos de risco e oportunidades para ao crescimento pessoal (STOCK NM, et al., 2015).

\section{Protocolos de tratamento}

A interdisciplinaridade é altamente recomendável, para enfrentamento das necessidades múltiplas do paciente com FLP e da família. A equipe deve incluir médicos, fonoaudiólogos, psicólogos, geneticistas, nutricionistas, dentistas, enfermeiros e outros profissionais (LEWIS CW, et al.,2017). Porém não há um consenso de tratamento entre os diferentes serviços pelo mundo e os protocolos podem variar (CHEPLA KJ e GOSAIN AK, 2013).

No Brasil, existem serviços especializados, como é o caso do Hospital de Reabilitação de Anomalias Craniofaciais da Universidade de São Paulo (HRAC-USP), cujo protocolo segundo FREITAS JAS, et al (2012) é descrito abaixo: A data da primeira visita do paciente ao hospital é recomendada no pré-natal ou logo nos primeiros dias após o nascimento. Nessa oportunidade, serão realizados esclarecimentos a respeito do diagnóstico, do manejo dos problemas associados à FLP e é delineado o tratamento ao longo prazo.

As primeiras cirurgias reparadoras são, usualmente, a queiloplastia realizada aos 3 meses de idade, e a palatoplastia aos 12 meses. As cirurgias reparadoras visam um equilíbrio entre o crescimento craniofacial e o desenvolvimento da fala do paciente com FLP. O objetivo da queiloplastia é a melhora da competência oral, da simetria e da estética. As técnicas mais utilizadas são as de Millard (fissuras bilaterais) ou de Fisher (unilaterais). 
Recomenda-se para a adesão do lábio, que o lactente pese no mínimo $4 \mathrm{Kg}$ e tenha nível de hemoglobina de $9,5 \mathrm{~g} / \mathrm{dl}$ ou mais, para menores riscos cirúrgicos. A palatoplastia, fecha a conexão entre as cavidades oral e nasal e reconstrói a musculatura palatina favorecendo o desenvolvimento da fala. Os riscos da cirurgia de palato incluem a formação de fístula, disfunção velofaríngea, distúrbios respiratórios do sono e distúrbios do crescimento da maxila (CHEPLA KJ e GOSAIN AK, 2013).

Ainda segundo o protocolo do HRAC-USP, aproximadamente aos 6 anos de idade, cirurgias secundárias para aprimoramento estético, funcional ou correção de possíveis fístulas de palato podem ser realizadas. $\mathrm{Na}$ adolescência, as correções nasais, como a rinosseptoplastia e as cirurgias ortognáticas podem ser necessárias. No período entre as cirurgias, efetua-se acompanhamento médico, o monitoramento da fala e da audição. Tratamento odontológico para minimizar as discrepâncias maxilo mandibulares e os problemas de dentição são realizados até o final da maturação esquelética. $\mathrm{Na}$ adolescência os cuidados ao paciente visam a melhora da oclusão e posicionamento dentários, a permeabilidade nasal, e as relações esqueléticas da face.

\section{CONSIDERAÇÕES FINAIS}

A FLP representa uma anomalia frequente, que requer tratamento especializado ao longo da vida do paciente. Apesar dos grandes avanços na compreensão dos problemas enfrentados pelos indivíduos com FLP, algumas questões permanecem pouco estudadas e merecem atenção, como é o caso das comorbidades manifestadas nos diferentes sistemas orgânicos. Os protocolos de tratamento podem variar em alguns aspectos entre diferentes serviços, mas a maioria dos autores ressaltam a importância da equipe interdisciplinar, do acompanhamento ao longo prazo e das pesquisas, para o manejo correto do paciente com FLP.

\section{REFERÊNCIAS}

1. BOYCE JO, et al. Academy of Breastfeeding Medicine ABM Clinical Protocol \#17: Guidelines for Breastfeeding Infants with Cleft Lip, Cleft Palate, or Cleft Lip and Palate-Revised2019. Breastfeed Med, 2019;14(7):437-444.

2. BRITTON KF, et al. An investigation into infant feeding in children born with a cleft lip and/or palate in the West of Scotland. Eur Arch Pediatr Dent, 2011;12(5):250-5.

3. BURG ML, et al. Epidemiology, Etiology, and Treatment of Isolated Cleft Palate. Front. Physiol, 2016;7:67.

4. CHRISTENSEN K, et al. Long term follow up study of survival associated with cleft lip and palate at birth. BMJ, 2004; 328(7453):1405.

5. CHEPLA KJ, GOSAIN AK. Evidence-based medicine: cleft palate. Plast Reconstr Surg, 2013;132(6):1644-1648.

6. DeROO LA, et al. Maternal alcohol binge-drinking in the first trimester and the risk of orofacial clefts in offspring: a large population based pooling study. Eur J Epidemiol, 2016;31(10):1021-1034.

7. DIXON MJ, et al. Cleft lip and palate: synthesizing genetic and environmental influences. Nat Rev Genet, 2011;12(3):167-178.

8. FERAGEN KB, et al. Toward a Reconsideration of Inclusion and Exclusion Criteria in Cleft Lip and Palate: Implications for Psychological Research. The Cleft Palate Craniofac J, 2014;51(5):569-578.

9. FREBOURG T, et al. Cleft lip/palate and $\mathrm{CDH} 1 / \mathrm{E}$-cadherin mutations in families with hereditary diffuse gastric cancer. J Med Genet, 2006;43:138-142.

10. FREITAS JAS, et al. Rehabilitative treatment of cleft lip and palate: experience of the Hospital for Rehabilitation of Craniofacial Anomalies/USP (HRAC/USP), Part 1: overall aspects. J Appl Oral Sci, 2012; 20:9-15

11. GILDESTAD T, et al. Folic acid supplements and risk for oral clefts in the newborn: a population-based study. $\mathrm{Br} \mathrm{J}$ Nutr, 2015;114(9):1456-1463.

12. GROSEN D, et al. A cohort study of recurrence patterns among more than 54,000 relatives of oral cleft cases in Denmark: support for the multifactorial threshold model of inheritance. J Med Genet, 2010;47(3):162-168.

13. HOSSEINABAD HH, et al. Incidence of velopharyngeal insufficiency and oronasal fistulae after cleft palate repair: A retrospective study of children referred to Isfahan Cleft Care Team between 2005 and 2009. Int J Pediatr Otorhinolaryngol, 2015;79(10):1722-1726.

14. HUNT O, et al. The psychosocial effects of cleft lip and palate: a systematic review. Eur J Orthod, 2005;27(3):274-85.

15. HUTCHINSON K, et al. The Psychosocial Effects of Cleft Lip and Palate in Non-Anglo Populations: A Cross-Cultural Meta-Analysis. Cleft Palate Craniofac J, 2011;48(5):497-508.

16. KUO CL, et al. Grommets for otitis media with effusion in children with cleft palate: a systematic review. Pediatrics, 2014;134(5):983-994.

17. LEWIS CW, et al. The Primary Care Pediatrician and the Care of Children with Cleft Lip and/or Cleft Palate. Pediatrics, 2017;139(5):e20170628.

18. MANGOLD E, et al. Genome-wide association study identifies two susceptibility loci for nonsyndromic cleft lip with or without cleft palate. Nat Genet, 2010; 42:24-26. 
19. MARQUES IL, et al. Longitudinal study of growth of children with unilateral cleft-lip palate from birth to two years of age. Cleft Palate Craniofac J, 2009;46(6):603-609.

20. MARTIN V, GREATREX-WHITE S. An evaluation of factors influencing feeding in babies with a cleft palate with and without a cleft lip. J Child Health Care, 2014;18(1):72-83.

21. MOSSEY PA, et al. Cleft lip and palate. Lancet, 2009; 374:1773-1785.

22. MURRAY J. Gene/environment causes of cleft lip and/or palate. Clin Genet, 2002;61:248-256.

23. NELSON PA, et al. Parents' experiences of caring for a child with a cleft lip and/or palate: a review of the literature. Child Care Health Dev, 2012;38(1):6-20.

24. REID J, et al. A prospective longitudinal study of feeding skills in a cohort of babies with cleft conditions. Cleft Palate Craniofac J, 2006;43(6):702-709.

25. ROTTEN D, LEVAILLANT JM. Two and three-dimensional sonographic assessment of the fetal face. 2 Analysis of cleft lip, alveolus and palate. Ultrasound in Obstet Gynecol, 2004;24(4): 402-411.

26. $\mathrm{SHI} \mathrm{M}$, et al. Orofacial cleft risk is increased with maternal smoking and specific detoxificationgene variants. Am $\mathrm{J}$ Hum Genet, 2007; 80(1):76-90.

27. SILVA HPV, et al. Risk factors and comorbidities in Brazilian patients with orofacial clefts. Braz. oral res, $2018 ; 32: \mathrm{e} 24$.

28. SOUSA GF, RONCALLI AG. Orofacial clefts in Brazil and surgical rehabilitation under the Brazilian National Health System. Braz. oral res. 2017;31(0):e23.

29. STOCK NM, et al. It Doesn't All Just Stop at 18": Psychological Adjustment and Support Needs of Adults Born With Cleft Lip and/or Palate. Cleft Palate Craniofac J, 2015;52(5):543-554.

30. TRINDADE IEK, SILVA FILHO OG. Fissuras labiopalatinas: uma abordagem interdisciplinar. São Paulo: Ed. Santos, 2007; 335p.

31. VOLK AS, et al. The History and Mission of Smile Train, a Global Cleft Charity. Oral Maxillofac Surg Clin North Am, 2020;32(3):481-488.

32. WEHBY G, et al. Oral cleft and behavioral health of young children. Oral Dis, 2012;18(1):74-84.

33. WEINBERG S, et al. Rethinking isolated cleft palate: Evidence of occult lip defects in a subset of cases. Am $\mathrm{J}$ Med Genet, 2008;146A:1670-1675.

34. ZEYTINOGLU S, Davey MP. It's a privilege to smile: impact of cleft lip palate on families. Fam Syst Health, 2012;30(3):265-277.

35. ZHU JL, et al. Do parents of children with congenital malformations have a higher cancer risk? A nationwide study in Denmark. Br J Cancer, 2002; 87:524-528. 